\title{
Possible Role of Antimatter Galaxies for the Stability of the Universe
}

\author{
S. Beghella-Bartoli, R. M. Santilli \\ Thunder Energies Corporation, 1444 Rainville Rd, Tarpon Springs, FL
}

Email address:

research@thunder-energies.com

\section{To cite this article:}

S. Beghella-Bartoli, R. M. Santilli. Possible Role of Antimatter Galaxies for the Stability of the Universe. American Journal of Modern Physics. Special Issue: Issue II: Foundations of Hadronic Mechanics. Vol. 5, No. 2-1, 2016, pp. 185-190.

doi: 10.11648/j.ajmp.2016050201.21

Received: January 19, 2016; Accepted: January 19, 2016; Published: June 1, 2016

\begin{abstract}
Recent mathematical, theoretical and experimental studies have confirmed via measurements on Earth Zwicky's hypothesis according to which the cosmological redshift is due to galactic light losing energy to intergalactic media without the expansion of the universe. The main problem of the ensuing return to a static universe is the inevitable prediction that the universe should collapse due to gravitational attractions among galaxies. In this paper, we review the historical inability by general relativity to achieve a stable universe solely composed of matter, and present apparently for the first time a cosmological model in which the universe achieves stability under the condition of admitting an equal number of matter and antimatter galaxies at such a large mutual distance for which gravitational interactions are ignorable.
\end{abstract}

Keywords: Antimatter, Static Universe, Universe Stability

\section{Historical Notes}

The study of the origin, evolution, and eventual fate of the universe has always been debated by philosophers and cosmologists, beginning with Aristarchus, Aristotle and Ptolemy. In particular, the geocentric theory of Ptolemy has been the dominant paradigm until it was put into question by Copernicus, Kepler, Galilei and Newton. But it was Albert Einstein, who essentially set up the foundations of what we nowadays call modern cosmology [1].

Given the relative very small velocities of stars in the Milky Way, the general belief in the early part of the 20th century was that we were living in a static universe. The main assumptions by Einstein were that there is a system of reference relatively to which matter may be looked upon as being permanently at rest, and that the large scale structure and evolution of such a universe should be determined by its finite matter density.

These assumptions implied a difficulty in the determination of border conditions at infinity, a difficulty that was solved by Einstein with the hypothesis of a closed universe without borders. But to achieve a finite matter density in a static universe, Einstein was forced to introduce the cosmological constant in his field equations for the intent of achieve stability via a repulsive force counter-balancing the gravitational attraction.

Einstein himself didn't like the addition of the cosmological constant in his equations because it looked to him just like an ad-hoc hypothesis that achieved the desired stability but without explaining how the universe works. Numerous additional doubts on the validity of the model were expressed, such as those by Willem de Sitter who suggested Einstein to get rid of the lambda-term in his field equations [2, 3]. In essence, de Sitter argued that the cosmological constant is arbitrary and detracts from the elegance of Einstein's original theory of gravitation.

De sitter also proposed a model that bypassed the problem of border conditions at infinity in which space is basically empty, with zero matter density, zero curvature and no division between time and space (unlike Einstein's model where an absolute time existed), thus achieving isotropy and homogeneity throughout space and time.

This solution was considered quite seriously by Einstein. De Sitter had used lambda to derive an empty model (so no finite density of matter) that actually satisfies boundary conditions at infinity. So the lambda-term went against Einstein's own beliefs (the field should be due to the matter, without which it cannot exist), and he persuaded himself he 
needed to get rid of it one way or the other.

With the passing of time, experimental measurements started to become available, with particular reference to the historical measurements by Hubble [4] on the redshift of light coming from distant "nebulas," later on called galaxies, according to the law $\mathrm{z}=\mathrm{Hd}$, where $\mathrm{z}$ is the redshift, $\mathrm{H}$ is the Hubble constant, and $\mathrm{d}$ is the distance of the galaxy from Earth. This view was embraced by Eddington [5], Weyl [6, 7], Slipher [8], Friedmann [9, 10], Lemaitre [11] and others.

It appears that, quite likely, Einstein's main desire was the removal of the cosmological constant from his field equations because his main concern was to account for a finite density of matter in the universe, and since it seemed he could do that via other models, he was happy to cast away what he thought was a troublesome addition to his theory [12]. This doesn't mean he approved the expansion and related Big Bang, but he didn't like the use of an arbitrary ad$h o c$ term to obtain equilibrium in a matter-only universe.

Einstein and de Sitter eventually came up with a new model, known as Einstein-de Sitter model [13], that influenced cosmology for the next 50 years. The solution implied a homogeneous and isotropic universe, with zero space curvature, zero cosmological constant, and zero pressure, with asymptotically null expansion (see for more details, e.g., Refs. [13-15].

The aspect most important for this paper is that, to our best understanding of historical profiles, Einstein never explicitly acknowledged the representation of the cosmological redshift via the conjecture of the expansion of the universe. Although he didn't participate actively to the cosmological debate after his 1917 paper, he continued to prefer a stationary universe, as it is clearly demonstrated by an unpublished paper of his [16], written in 1931 and recently rediscovered [17, 18], where, after Hubble's paper, he developed another steadystate model of the universe keeping into account Hubble's results, but, considering a pure matter universe, he apparently faced mathematical inconsistencies. The same position was adopted by Hubble and other cosmologists, such as Fritz Zwicky [19] who proposed the hypothesis that the redshift of galactic light is due to loss of energy by light to inter-galactic gases.

In summary, during the first part of the 20th century, jointly with the initiation of studies on the interpretation of Hubble's law via the expansion of the universe, there were also authoritative views that essentially implied a static conception of the universe, mostly according to Hoyle's cosmology [20]. It is important to note that Einstein himself basically anticipated Hoyle's view in his 1931 unpublished paper.

\section{Recent Debates in Cosmology}

Even today, after so many years the debate is still open, with the so-called Standard model suffering of multiple flaws, and many scientists looking back at models of the universe that don't need a Big Bang, if not completely static.

On May 22, 2004, an open letter was signed by many scientists and published in New Scientist [21]. In this letter they complained about the many inconsistencies of the Big Bang theory and its corollaries, stating that "the successes claimed by the Big Bang theory's supporters consist of its ability to retrospectively fit observations with a steadily increasing array of adjustable parameters, just as the old Earth-centered cosmology of Ptolemy needed layer upon layer of epicycles. Yet the big bang is not the only framework available for understanding the history of the universe. Plasma cosmology and the steady-state model both hypothesize an evolving universe without beginning or end. These and other alternative approaches can also explain the basic phenomena of the cosmos."

Since publication, the letter has been signed by more than 500 researchers worldwide.

A group of those researchers created an association called "Alternative Cosmology Group" [22], that, as stated in their website, "is an open society of scientists from all over the world, dedicated to the advance in cosmology and basic research". In this site it's possible to find a wide variety of papers and articles presenting both observational results and theoretical research suggesting an alternative point of view on the evolution and fate of our universe.

As an example, in one of the most recent articles [23], the authors compared the size and brightness of many galaxies at different distances from us, considering the most luminous spiral galaxies for comparisons, finding that, contrary to the prediction of the Big Bang theory, near and far galaxies have similar surface brightness. The authors "conclude that available observations of galactic $S B$ (surface brightness) are consistent with a static Euclidean model of the universe", therefore "the redshift is due to some physical process other than expansion".

It may be also interesting for the purposes of this paper to mention some other researchers that, even in the "mainstream" interpretation of measured redshifts, thus considering an expanding universe, completely disagree with the idea of a Big Bang and consequent dark energy and matter, and find in the repulsive gravity of antimatter a possible solution of the inconsistencies met by Einstein and others. This is the case of Dr. Massimo Villata of the Italian National Institute for Astrophysics (INAF) and Dr. Dragan Hajdukovic, physicist at CERN.

The first one has developed a theory according to which repulsive gravity between matter and antimatter located in cosmic voids (and, important to stress, not detectable by our standard instruments) can account for universe expansion without need of dark energy or matter and Big Bang.

It is interesting to notice also that according to his calculations a reasonable antimatter mass, located in a particular void, could account for a recorded local velocity anomaly of the "Local Sheet," the part of the universe that includes the Milky Way and other nearby galaxies, by the mechanism of repulsive gravity. This local anomaly apparently couldn't be explained by a "dark energy" that acts uniformly throughout the space $[24,25]$. As the author states "Through simple dynamical considerations, we find that the 
Local Void could host an amount of antimatter roughly equivalent to the mass of a typical supercluster, thus restoring the matter-antimatter symmetry. Like matter, antimatter is self-attractive, so we can expect that it forms anti-galaxies and anti-stars, which would emit electromagnetic radiation, we could then detect. However antimatter, if emitting, should emit advanced radiation, which can be undetectable".

The second researcher also considers a repulsive gravitational interaction between matter and antimatter as an alternative to dark energy, dark matter and Big Bang, but he focuses on the microscopic interactions at the particle level. In his view repulsive gravity of virtual particles and antiparticles in quantum vacuum can explain several observations, including effects usually attributed to dark matter [26].

\section{The Zwicky-Santilli Effect}

The historical accounts outlined in the preceding section indicate that Einstein, Hubble, Zwicky, Hoyle, and other cosmologists died without accepting the conjecture of the expansion of the universe apparently because Hubble's law z $=$ Hd clearly establishes the same redshift for all galaxies at the same distance $\mathrm{d}$, in all possible radial directions from Earth, thus essentially implying a return to a Ptolemaic view of the universe (Fig. 1).

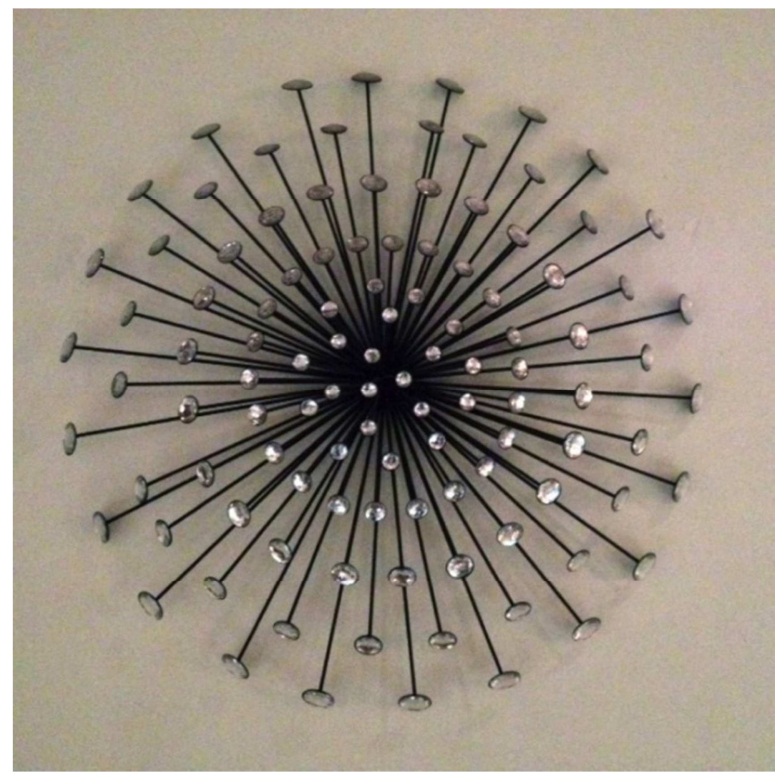

Figure 1. A sculpture illustrating the radial character of the interpretation of the Hubble Law $z=H d$, via the expansion of the universe with an evident return to the Ptolemaic conception of Earth at the center of the universe.

In order to honor the above view, one of us (R. M. Santilli) conducted decades of mathematical, theoretical and experimental studies that have confirmed Zwicky's hypothesis via measurements on Earth resulting in an effect known as the Zwicky-Santilli effect (for brevity, see the collection of references [27]).

It may be interesting to mention that studies [27] are complemented by the suggestive possibility of a continuous creation of matter in the universe occurring in the core of stars during their synthesis of the neutron from the hydrogen atom [28].

\section{The Proposed Isoselfdual Universe}

The main problem for a return to a static universe is its inherent prediction that the universe should collapse due to gravitational attractions between galaxies.

By remembering from Section 1 that could not be resolved via theories for matter, in this paper, we present apparently for the first time a cosmological model achieving stability of the universe via an appropriate distribution of matter and antimatter galaxies.

To avoid a prohibitive length, the understanding of the proposed model requires a knowledge of: the isodual theory for the classical representation of neutral or charged antimatter [29]; the prediction of matter-antimatter gravitational repulsion occurring at all levels of study, the recent apparent detection of antimatter galaxies via telescopes with concave lenses and the recent apparent detections of antimatter galaxies, antimatter asteroids, and antimatter cosmic rays (for brevity, see list [30] referred publications in the field).

Let us consider a distribution of point-like matter and antimatter masses in vacuum only subject to gravitational interactions. We can then study the stability of this simplified system in order to appraise the correctness of our hypothesis. As further simplification, we first consider masses in a onedimensional space, and then extend the model to three dimensions.

In order to look for an equilibrium of the system, we have to study the first derivative of the state vector, which is characterized by position and velocity of the point masses. The derivative of position at the initial time is assumed to be zero, since masses are assumed to be initially at rest, so the only significant component to look at is the derivative of velocity.

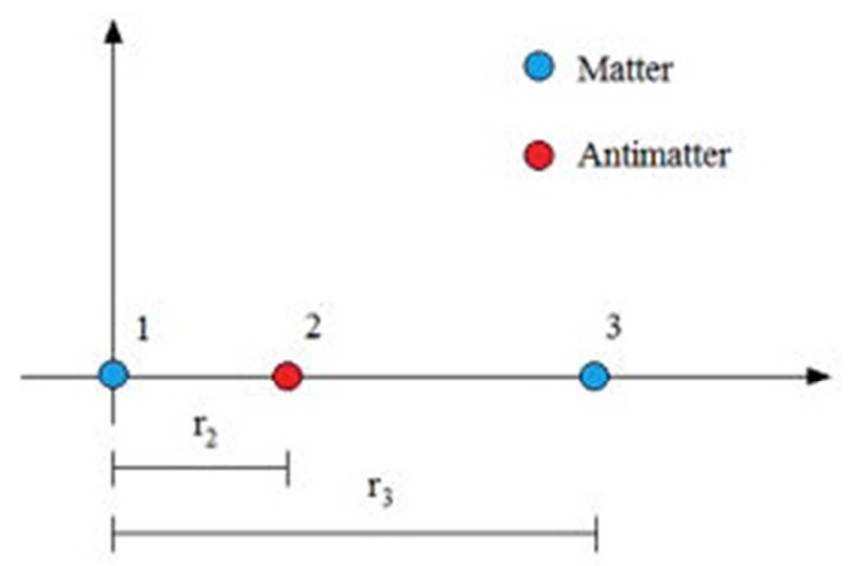

Figure 2. We illustrate the case of three unit masses in a one-dimensional, according to the distribution of one matter mass, one antimatter mass and one matter mass under the assumption of matter-antimatter gravitational repulsion. 
Considering all possible permutations of up to 12 masses (half of them matter and half antimatter) at uniform finite distances, we easily find out that they are never in equilibrium, because the resultant acceleration on each mass is always different than zero. The use of variable distances for the same masses shows that equilibrium can be achieved only when distances themselves tend to infinity.

To better investigate the latter aspect, we study the equilibrium of 3 masses only (Fig 2), so that we can plot the results in a single graph. Being in one-dimension, there are only 2 free coordinates, which are the positions of the second and third mass with respect to the first one, and the accelerations can be plotted as a 3-D surface (Fig 3).
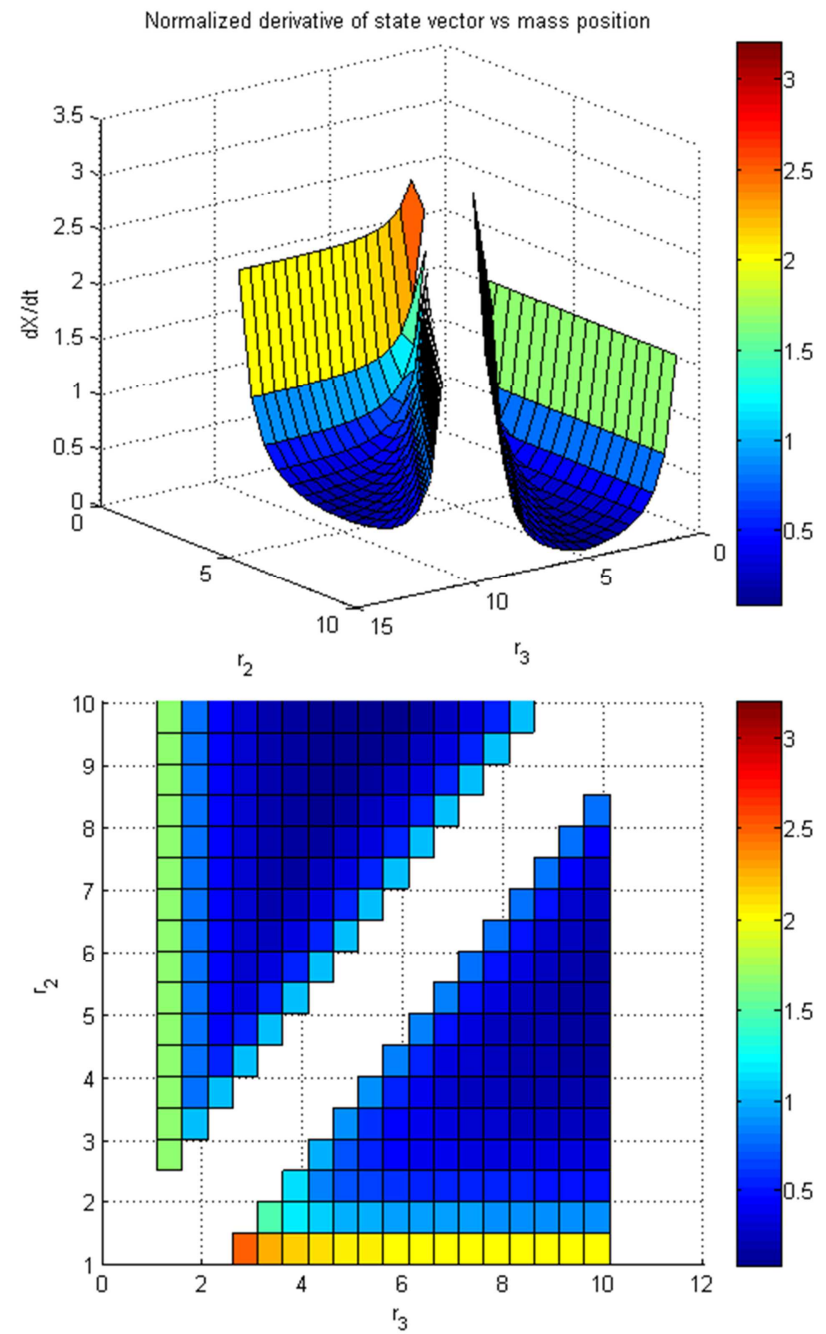

Figure 3. The graph shows the normalized gravity acceleration acting on the three unit masses in the one-dimensional case of Figure 2 versus the positions of the masses. The values shown are the sum of absolute values of the resultant accelerations acting on each mass, due only to gravitational interactions among the masses themselves. From the graph it is possible to see that the global acceleration never crosses the zero plane, but it tends to decrease asymptotically with the increasing of the distances. In the diagonal and at the borders the acceleration tends to infinity because in these cases the positions of two of the masses tend to coincide. We have the lowest accelerations in the blue parts of the graph, where the masses tend to have large equal distances.

As we can see from Figures 3 to 5 , the resultant accelerations between matter and antimatter decreases with the increase of their distance, and reach equilibrium at a mutual distance for which the gravitational repulsion is ignorable.

Therefore, for better readability, we did plot also the accelerations in the case of equidistant masses in the sequence matter-antimatter-matter (Fig 4). Having only one variable, the resulting graph (Fig 5) is two-dimensional, and it clearly shows that the accelerations decrease with the increasing of distance, tending asymptotically to zero at infinity.

Needless to say, the proposed cosmological model needs considerable additional studies. However, it is rather easy to show that the extension of the model to two and three dimensions readily the same results.

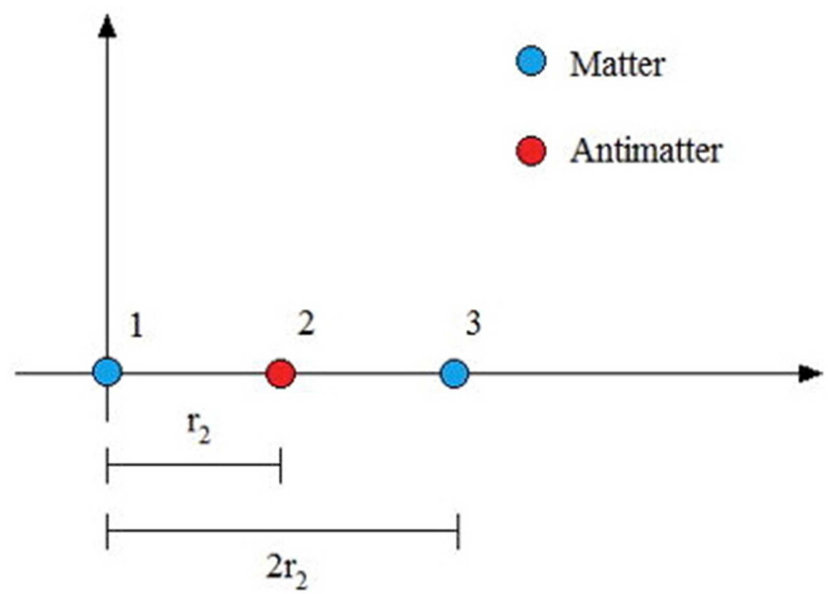

Figure 4. In this case the three $u$ unit masses are considered again in onedimension, but they are equidistant.

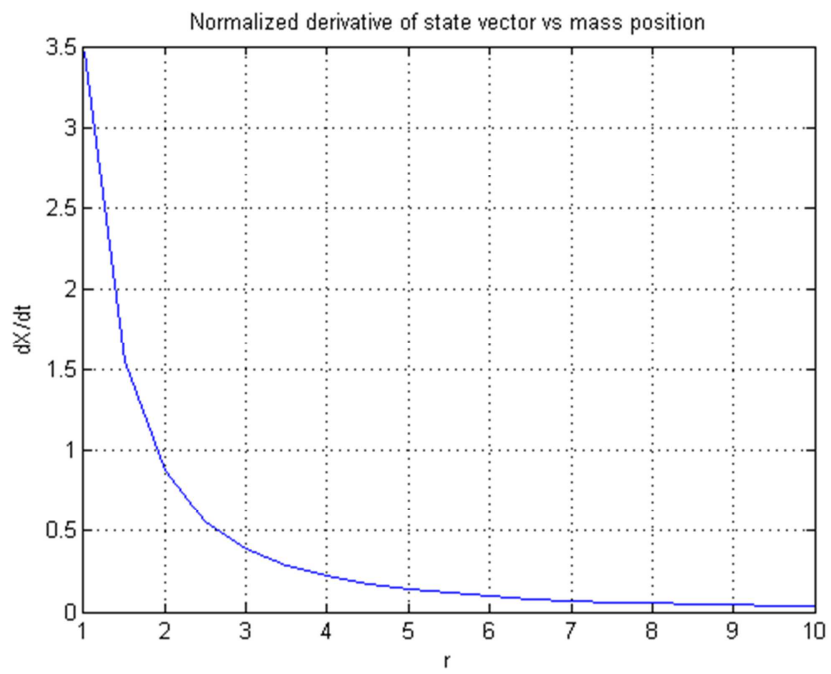

Figure 5. The graph shows the normalized gravity acceleration acting on the three equidistant unit masses with the sequence matter-antimatter-matter in one-dimension versus the positions of the masses themselves. We know from the previous graph that in this case the acceleration tends to be a minimum. From this figure, it is possible to see more clearly that the sum of absolute values of accelerations tends asymptotically to zero with the increasing of the distances among the masses themselves. 


\section{Conclusions}

By remembering from Section 1 that the stability of the universe when solely composed by matter could not be consistently achieved via general relativity or other theories, we can conclude by saying that the universe can indeed achieve stability under the conditions of being composed by an equal number of matter and antimatter galaxies having a null total mass, matter and antimatter galaxies being at such large mutual distances caused by their gravitational repulsion at which all gravitational interactions are ignorable.

Needless to say, the above conditions merely represent limit values with large possibilities of local variations, such as: individual pairs of matter and antimatter galaxies need not necessarily have a null total mass since the gravitational repulsion exists also for different masses in absolute value; the conditions that the gravitational repulsion between matter and antimatter galaxies is ignorable implies very large variations of relative distances as well as (moderate) speeds, etc.

On more advanced grounds, we should note that the proposed cosmological model is isoselfdual, in the sense that it coincides with its isodual image [29], thus verifying the strict invariance under Santilli isoselfdual symmetry given by the Poincare' symmetry times its isodual [29]. The most rigorous proof of the consistency of our cosmological model (we can now call isoselfdual cosmology) can then be proved via the invariance under Santilli isoselfdual symmetry.

Recall that, in order to achieve a classical representation of neutral antimatter, it resulted necessary to assume that all characteristics of antimatter are opposite those of matter. Consequently, it is suggestive to note that all physical characteristics of the universe are identically null in our isoselfdual cosmology, thus including null total time, null total energy, etc.

The latter feature appears intriguing for quantitative studies on the origin of the universe without all calculations being lost at the act of creation due to singularities, since in the isoselfdual cosmology there is no change of numerical value of total physical quantities before and after creation. In turn, such a feature appears to support the suggestive hypothesis of a continuous creation in the core of matter and antimatter stars [28].

\section{References}

[1] Einstein, A. 1917. "Kosmologische betrachtungen zur allgemeinen relativit"atstheorie." Sitzungsber., K. Preuss. Akad. Wiss. 142-152. English translation in H. A. Lorentz, et al., eds. 1952. "The principle of relativity". Dover Publications, Mineola, New York, 175-188.

[2] De Sitter, W. 1917a. "On the relativity of inertia. Remarks concerning Einsteins latest hypothesis.", Proc. R. Acad. Amsterdam 1217-1225.

[3] De Sitter, W. 1917b. "Einsteins theory of gravitation and its astronomical consequences." Third paper. Mon. Not. R. Astron. Soc. 78: 3-28.
[4] Hubble, E. 1929. "A relation between distance and radial velocity among extra-galactic nebulae.", Proc. Nat. Acad. Sci. 15: 168-173.

[5] Eddington, A. S. 1923. "The mathematical theory of relativity", 2nd ed. Cambridge University Press, Cambridge.

[6] Weyl, H. 1923. "Raum, zeit, materie: vorlesungen über allgemeine relativit"atstheorie, funft mgearbeitete auflage." Julius Springer, Berlin.

[7] Weyl, H. 1923. "Zur allgemeinen relativit"atstheorie." Phys. Z. 24: 230-232 (1923b). English translation: Weyl, H. 2009. "Republication of: On the general relativity theory". Gen. Relativ. Gravitat. 35: 1661-1666.

[8] Slipher, V. M., 1913, "The radial velocity of the Andromeda Nebula." Lowell Observatory Bulletin 2: 56-57.

[9] Friedmann, A. 1922: “'Uber die Kr"ummung des Raumes.” Z. Phys. 10: 377-386. English translation: Friedman, A. "On the curvature of space.” Gen. Rel. Gravitat. 31: 1991-2000.

[10] Friedmann, A. 1924. "“Uber die M"oglichkeit einer Welt mit konstanter negativer Kr ummung des Raumes." Z. Phys. 21: 326-332. English translation: Friedmann, A. "On the possibility of a world with constant negative curvature of space.” Gen. Rel. Gravitat. 31 2001-2008.

[11] Lemaître, G. 1927. "Un Univers homogène de masse constante et de rayon croissant rendant compte de la vitesse radiale des nbuleuses extra-galactiques." Annales de la Société Scientifique de Bruxelles A, 47: 49-59. English translation: Lemaître, G. 1931. "A Homogeneous universe of Constant Mass and Increasing Radius accounting for the Radial Velocity of Extra-Galactic Nebulae." Mon. Not. R. Astron. Soc. 91: 483-490.

[12] Einstein, A. 1931. Letter to R. Tolman, Einstein Archives call no. 23-30. Post-dated June 27, 1931.

[13] Einstein, A. and W. de Sitter. 1932 "On the relation between the expansion and the mean density of the universe" Proc. Nat. Acad. Sci. 18: 213-214.

[14] P. Kerszberg, 1989, "The Einstein - de Sitter controversy of 1916-1917 and the rise of relativistic cosmology", Einstein and the history of general relativity, p. 325-366.

[15] D. Janzen, 2016 "Einstein's cosmological considerations", 53 pages. Submitted to Eur Phy J (H), arXiv: 1402.3212.

[16] Einstein, A. 1931. Zum kosmologischen Problem, Einstein Archives call no. 23-30.

[17] C. O'Raifeartaigh and B. McCann, "Einstein's cosmic model of 1931 revisited: an analysis and translation of a forgotten model of the universe", European Physical Journal H 39 (2014) 63-85. DOI: 10.1140/epjh/e2013-40038-x and arXiv: 1312.2192.

[18] Nature 506, 418-419 (27 February 2014).

[19] Zwicky, F., 1929, Proceedings of the National Academy of Sciences of the United States of America, 773.

[20] Hoyle, F., 1948 Mon. Not. R. Astron. Soc. 108, 372-382.

[21] VV. AA., "Open Letter on Cosmology", New Scientist, issue 2448 May 22, 2004 http://homepages.xnet.co.nz/ hardy/cosmologystatement.html.

[22] http://www.cosmology.info/. 
[23] Eric J. Lerner, Renato Falomo, and Riccardo Scarpa, "UV surface brightness of galaxies from the local universe to $\mathrm{z} \sim 5$ ", International Journal of Modern Physics D Vol. 23, No. 6 (2014) 1450058 DOI: 10.1142/S0218271814500588.

[24] Massimo Villata. "'Dark Energy' in the Local Void." Astrophysics and Space Science (2012) 339: 7-12. DOI: 10.1007/s10509-012-0994-9 and arXiv: 1201.3810v1 [astroph. CO].

[25] Massimo Villata. "CPT symmetry and antimatter gravity in general relativity", EPL (Europhysics Letters), Volume 94, Number 2, 2011.

[26] Dragan Hajdukovic. "Quantum vacuum and virtual gravitational dipoles: the solution to the dark energy problem?" Astrophysics and Space Science. DOI: 10.1007/s10509-0120992-y.
[27] Fleming, 2014, P. Collected papers, interviews, seminars and international press releases on the lack of expansion of the universe, http://www.santilli-foundation.org/docs/Nouniverse-expans.pdf.

[28] Santilli, R. M., 2007, "The etherino and/or the neutrino hypothesis," Foundation of Physics $<\mathrm{b}>37</ \mathrm{b}>, \quad 670$, http://www.santillifoundation.org/docs/EtherinoFoundPhys.pdf.

[29] R. M. Santilli, 2005, Isodual Theory of Antimatter with Applications to Antigravity, Grand Unifications and Cosmology, $\quad$ Springer, http://www.santillifoundation.org/docs/santilli-79.pdf.

[30] Fleming, 222015, P. Scientific references and PR Web News Releases on $\backslash$ Santilli isodual Theory of Antimatter http://www.santilli-foundation.org/docs/Santilli-TelescopeRefs-1-15.p. 